Calcolo 43: 197-215, 2006

\title{
A FINITE ELEMENT MODEL FOR MARTENSITIC THIN FILMS
}

\author{
PAVEL BĚLÍK AND MITCHELL LUSKIN
}

\begin{abstract}
A finite element approximation of the thin film limit for a sharp interface bulk energy for martensitic crystals is given. The energy density models the softening of the elastic modulus controlling the low-energy path from the cubic to the tetragonal lattice, the loss of stability of the tetragonal phase at high temperatures and the loss of stability of the cubic phase at low temperatures, and the effect of compositional fluctuation on the transformation temperature. The finite element approximation is then used to simulate the hysteresis of a martensitic thin film obtained after applying a biaxial loading cycle to the film below the transformation temperature.
\end{abstract}

\section{INTRODUCTION}

We present a finite element approximation of the $\Gamma$-limit dimensional reduction of a threedimensional bulk energy for the deformation of a martensitic crystal $[3,4,6-8,13,16,19,20,25-29$, $31-34,37,40-43]$. The rigorously derived thin film model $[11,12,15]$ is more general than previously considered and includes transverse shear, normal compression, and biaxial loading. The free energy density models the softening of the elastic modulus controlling the low-energy path from the cubic to the tetragonal lattice, the loss of stability of the tetragonal phase at high temperatures and the loss of stability of the cubic phase at low temperatures, and the effect of compositional fluctuation on the transformation temperature $[13,14,24]$.

We use this finite element approximation to simulate the hysteresis of the structural phase transformation in a single-crystal film that undergoes a biaxial loading cycle $[1,5]$ in the low temperature phase.

We describe the bulk energy in Section 2, the loading cycle in Section 3, the thin film model in Section 4, the finite element approximatin in Section 5, the elastic energy density in Section 6, the numerical experiment in Section 7, and the numerical results and their interpretation in Section 8.

\section{The Bulk Energy}

We will assume that the deformation $u: \Omega_{h} \rightarrow \mathbb{R}^{3}$ of a martensitic film of thickness $h>0$ with reference configuration $\Omega_{h} \equiv \mathcal{S} \times(-h / 2, h / 2) \subset \mathbb{R}^{3}$ for $\mathcal{S}=(-1 / 2,1 / 2) \times(-1 / 2,1 / 2)$ quasistatically evolves as the temperature $\theta$ and boundary load are varied. We model the total free energy for the deformation $u: \Omega_{h} \rightarrow \mathbb{R}^{3}$ by the sum of its interfacial energy, elastic energy, and loading potential energy

$$
\mathcal{E}_{h}(u ; \theta, \sigma)=\kappa \int_{\Omega_{h}}|D(\nabla u)|+\int_{\Omega_{h}} \phi(\nabla u(x), \theta, c(x)) d x-\int_{\partial \Omega_{h}} \sigma n \cdot u d S,
$$

where $\kappa>0$ is a measure of interfacial energy per unit area, $\phi(F, \theta, c)$ is a composition-dependent free energy density, $c(x)$ is a fixed composition for the alloy, and $\sigma n$ is the boundary load where

Date: July 1, 2005.

2000 Mathematics Subject Classification. 65C30, 65Z05, 74K35, 74N10, 74N15, 74S05.

Key words and phrases. finite element, phase transformation, martensite, austenite, thin film.

This work was supported in part by NSF DMS-0074043 and DMS-0304326, the Institute for Mathematics and Its Applications, and by the Minnesota Supercomputer Institute. 
$\sigma \in \mathbb{R}^{3 \times 3}$ and $n \in \mathbb{R}^{3}$ is the unit exterior normal to the boundary, $\partial \Omega_{h}$. The total variation of the deformation gradient, $\int_{\Omega_{h}}|D(\nabla u)|$, is defined in Section 4.

\section{The Loading Cycle}

We start the simulation with the film in the cubic (high temperature) reference state

$$
u(x)=x \quad \text { for all } x \in \Omega_{h} .
$$

We then compute the quasi-static cooling of the film from $315 \mathrm{~K}$ through the temperature at which the cubic phase loses stability and the tetragonal (low-temperature) phase becomes stable to $215 \mathrm{~K}$. These transformation temperatures and other moduli used in this paper were chosen to fit experimental data for $\mathrm{Fe}_{70} \mathrm{Pd}_{30}$ and are more fully explained in Section 6 .

At $\theta=215 \mathrm{~K}$, we apply a loading cycle for $\sigma \in \mathbb{R}^{3 \times 3}$ having the biaxial form

$$
\sigma=\left(\begin{array}{ccc}
\sigma_{1} & 0 & 0 \\
0 & \sigma_{2} & 0 \\
0 & 0 & 0
\end{array}\right),
$$

so $\sigma_{1}$ is the normal tensile load on the edges $x_{1}=-1 / 2$ and $x_{1}=1 / 2$, and $\sigma_{2}$ is the normal tensile load on the edges $x_{2}=-1 / 2$ and $x_{2}=1 / 2$. The loading cycle is:

(1) Uniaxial loading: $\sigma_{1}$ increases from $0.0 \mathrm{GPa}$ to $0.3 \mathrm{GPa}$ with $\sigma_{2}=0.0 \mathrm{GPa}$.

(2) Biaxial loading cycle: For $\sigma_{1}+\sigma_{2}=0.3 \mathrm{GPa}$,

(a) $\sigma_{1}$ decreases from $0.3 \mathrm{GPa}$ to $0.0 \mathrm{GPa}$, then

(b) $\sigma_{1}$ increases from $0.0 \mathrm{GPa}$ to $0.3 \mathrm{GPa}$.

(3) Unaxial unloading: $\sigma_{1}$ decreases from $0.3 \mathrm{GPa}$ to $0.0 \mathrm{GPa}$ with $\sigma_{2}=0.0 \mathrm{GPa}$.

We will see in Figure 1 and Table 1 that the volume fraction of the "red" variant increases during the uniaxial loading with our computational model. The volume fraction of the "red" variant decreases and the volume fraction of the "yellow" variant increases during the first stage of the biaxial loading cycle as $\sigma_{1}$ is decreased and $\sigma_{2}$ is increased, and the volume fraction of the "red" variant increases and the volume fraction of the "yellow" variant decreases during the second stage of the biaxial loading cycle as $\sigma_{1}$ is increased and $\sigma_{2}$ is decreased. The film then remains nearly entirely in the "red" variant during the subsequent unloading.

After the completion of the loading cycle, the film is first heated from $215 \mathrm{~K}$ to $315 \mathrm{~K}$, cooled from $315 \mathrm{~K}$ to $215 \mathrm{~K}$, heated again from $215 \mathrm{~K}$ to $315 \mathrm{~K}$, and then finally cooled from $315 \mathrm{~K}$ to $215 \mathrm{~K}$. We observe in Figures 2-3 and Tables 2-3 that the film returns to the "red" variant after each heating and cooling cycle.

We derive a numerical approximation by first replacing the bulk energy (2.1) by its thin film $\Gamma$-limit $[12,15]$ and by then introducing a finite element approximation. We then discretize the temperature and loading cycles described above, and to advance the continuation in temperature or load we compute a local minimum of the energy by the Polak-Ribière conjugate gradient method with initial iterate given by the previous state.

\section{The Thin Film Model}

We will use the free energy density for martensitic crystals with softening and compositional fluctuation introduced in [14,24]. This free energy density $\phi(F, \theta, c)$ is a continuous function $\phi$ : $\mathbb{R}_{+}^{3 \times 3} \times\left(\theta_{0}, \theta_{1}\right) \times[0,1] \rightarrow \mathbb{R}$ of the deformation gradient $F \in \mathbb{R}_{+}^{3 \times 3}$ (where $\mathbb{R}_{+}^{3 \times 3}$ denotes the set of $3 \times 3$ matrices with positive determinant), the temperature $\theta \in\left(\theta_{0}, \theta_{1}\right)$, and a compositional order parameter $c \in[0,1]$. We assume that the free energy density, $\phi=\phi(F, \theta, c)$, is frame indifferent and has the material symmetry of the cubic (austenitic) crystalline phase. Since the transformation 
temperature, $\theta_{T}$, depends monotonically and continuously on the composition, $c$, in the remainder of this paper we will denote the energy density as a function of transformation temperature, $\theta_{T}$, rather than composition, $c$; and we use the notation $\phi=\phi\left(F, \theta, \theta_{T}\right)$ for $F \in \mathbb{R}_{+}^{3 \times 3}$ and $\theta, \theta_{T} \in\left(\theta_{0}, \theta_{1}\right)$.

We assume that the function $\theta_{T}: \Omega_{1} \rightarrow\left(\theta_{0}, \theta_{1}\right)$ is fixed as the temperature and load are varied and satisfies the Carathéodory condition $[15,18,35]$

(1) $\theta_{T}\left(\hat{x}, x_{3}\right)$ is continuous in $x_{3} \in(-1 / 2,1 / 2)$ for almost every $\hat{x} \in S$,

(2) $\theta_{T}\left(\hat{x}, x_{3}\right)$ is measurable in $\hat{x} \in S$ for every $x_{3} \in(-1 / 2,1 / 2)$.

In the bulk free energy (2.1), the interfacial energy in a layer separating a region between phases and variants is proportional to the product of the surface area of the interface and the magnitude of the jump in the deformation gradient. We denote this interfacial energy by $\kappa \int_{\Omega_{h}}|D(\nabla u)|$, where the total variation of the deformation gradient $[11,12,21,23]$ is given by

$$
\begin{aligned}
\int_{\Omega_{h}} \mid & D(\nabla u) \mid \\
\quad & \sup \left\{\sum_{i, j, k=1,2,3} \int_{\Omega_{h}} u_{i, j}(x) \psi_{i j k, k}(x) d x: \psi \in \mathcal{C}_{0}^{\infty}\left(\Omega_{h} ; \mathbb{R}^{3 \times 3 \times 3}\right),|\psi(x)| \leq 1 \text { for all } x \in \Omega_{h}\right\}
\end{aligned}
$$

We use the usual euclidean norm, the square root of the sum of the squares of all the components, for the above norm, $|\cdot|$. With this definition, the total variation of a piecewise smooth deformation gradient $\nabla u$ that has jumps $\llbracket \nabla u \rrbracket_{\sigma_{j}}$ across the piecewise smooth surfaces $\sigma_{j}, j=1, \ldots, J$, separating the open sets $\omega_{\ell}$ in a disjoint union $\Omega_{h}=\sum_{\ell=1}^{L} \omega_{\ell}$ is given by

$$
\int_{\Omega_{h}}|D(\nabla u)|=\sum_{j=1}^{J} \int_{\sigma_{j}}\left|\llbracket \nabla u \rrbracket_{\sigma_{j}}\right| d S+\sum_{\ell=1}^{L} \int_{\omega_{\ell}} \sqrt{\sum_{m, n=1}^{3}\left(\frac{\partial^{2} u}{\partial x_{m} \partial x_{n}}\right)^{2}} d x .
$$

Our analysis of the $\Gamma$-limit of the bulk energy $(2.1)$ as $h \rightarrow 0[12,15]$ assumed that the energy density $\phi\left(F, \theta, \theta_{T}\right)$ satisfies the growth condition

$$
c_{1}|F|^{p}-c_{2} \leq \phi\left(F, \theta, \theta_{T}\right) \leq c_{3}\left(|F|^{p}+1\right) \quad \text { for all } F \in \mathbb{R}^{3 \times 3} \text { and } \theta, \theta_{T} \in\left(\theta_{0}, \theta_{1}\right),
$$

where $c_{1}, c_{2}$, and $c_{3}$ are fixed positive constants, and $p>3$ to ensure that deformations with finite energy are uniformly continuous $[2,22]$. (We note that our analysis of the $\Gamma$-limit in $[12,15]$ used the assumption that $\phi\left(F, \theta, \theta_{T}\right)$ is defined for all $F \in \mathbb{R}^{3 \times 3}$ rather than only for $F \in \mathbb{R}_{+}^{3 \times 3}$. The equations defining $\phi\left(F, \theta, \theta_{T}\right)$ in Section 6 can be used for all $F \in \mathbb{R}^{3 \times 3}$, although the deformation gradient in all of the computations reported in this paper had positive determinate everywhere.) Since $\sigma$ is assumed constant in space, we have by the divergence theorem that

$$
\mathcal{E}_{h}(u ; \theta, \sigma)=\kappa \int_{\Omega_{h}}|D(\nabla u)|+\int_{\Omega_{h}} \phi\left(\nabla u(x), \theta, \theta_{T}(x)\right) d x-\int_{\Omega_{h}} \sigma \cdot \nabla u(x) d x,
$$

for $u$ in the space of admissible deformations

$$
\overline{\mathcal{A}}_{1}=\left\{u \in W^{1, p}\left(\Omega_{1} ; \mathbb{R}^{3}\right): \nabla u \in B V\left(\Omega_{1}\right), \int_{\Omega_{1}} u=0\right\} .
$$

We showed in $[12,15]$ that the $\Gamma$-limit of $(4.2)$ scaled by the thickness, $\mathcal{E}_{h}(u ; \theta, \sigma) / h$, is given by

$$
\overline{\mathcal{E}}^{(0)}(y, b ; \theta, \sigma)=\kappa \int_{\mathcal{S}}|D(\nabla y \mid \sqrt{2} b)|+\int_{\mathcal{S}} \phi\left(\nabla y(\tilde{x}) \mid b(\tilde{x}), \theta, \theta_{T}(\tilde{x}, 0) d \tilde{x}-\int_{\mathcal{S}} \sigma \cdot(\nabla y(\tilde{x}) \mid b(\tilde{x})) d \tilde{x}\right.
$$


for pairs $(\tilde{y}, \tilde{b}) \in \overline{\mathcal{A}}_{0}$, where the space of admissible deformations of the thin film is given by

$$
\overline{\mathcal{A}}_{0}=\left\{(y, b) \in W^{1, p}\left(S ; \mathbb{R}^{3}\right) \times L^{p}\left(S ; \mathbb{R}^{3}\right): \nabla y, b \in B V(S), \int_{S} y=0\right\} .
$$

The "deformation gradient" matrix $(\nabla y \mid b) \in \mathbb{R}^{3 \times 3}$ has $\nabla y$ for its first two columns and $b$ as its last column. The "integral" $\int_{\mathcal{S}}|D(\nabla y \mid \sqrt{2} b)|$ is the total variation of the vector-valued function $(\nabla y \mid \sqrt{2} b): \mathcal{S} \rightarrow \mathbb{R}^{3 \times 3}$. The map $y: \mathcal{S} \rightarrow \mathbb{R}^{3}$ gives the deformation of the midplane of the film, and the map $b: \mathcal{S} \rightarrow \mathbb{R}^{3}$ gives the deformation of the cross-section relative to the film $[9,11,12]$.

Since we will only be concerned with the thin film model in the remainder of this paper, we will use the notation $\theta_{T}(\hat{x})$ for $\theta_{T}(\hat{x}, 0)$.

\section{Finite Element Approximation}

We denote a triangulation of $\mathcal{S}$ by $\tau$ with its triangular elements denoted by $K$. We define the jump of a piecewise constant function $\psi: \mathcal{S} \rightarrow \mathbb{R}^{3 \times 3}$ across an inter-element edge $e$ separating two elements $K_{1}, K_{2} \in \tau$ by

$$
\llbracket \psi \rrbracket_{e}=\psi_{e, K_{1}}-\psi_{e, K_{2}},
$$

where $\psi_{e, K_{i}}$ denotes the trace on $e$ of $\left.\psi\right|_{K_{i}}$ for $i=1,2$. We note that we only use the norm of the jump in what follows, so there is no ambiguity caused by the sign of the jump.

To develop the finite element approximation, we constrain the deformation $y(\tilde{x})$ to lie in $\overline{\mathcal{P}}_{1}(\tau)$, the space of continuous functions on $\mathcal{S}$ with mean value zero, $\int_{S} y=0$, which are linear on each $K \in \tau$, and we constrain the midplane strain $b(\tilde{x})$ to lie in $\mathcal{P}_{0}(\tau)$, the space of functions on $\mathcal{S}$ which are constant on each $K \in \tau$. The space of approximate admissible functions is then given by

$$
\overline{\mathcal{A}}_{\tau}=\overline{\mathcal{P}}_{1}(\tau) \times \mathcal{P}_{0}(\tau) \subset \overline{\mathcal{A}}_{0} .
$$

For $(y, b) \in \overline{\mathcal{A}}_{\tau}$ and $\theta, \theta_{T} \in \mathcal{P}_{0}(\tau)$, the energy (4.3) is given by

$$
\begin{aligned}
\kappa \int_{\mathcal{S}} \mid & D(\nabla y \mid \sqrt{2} b) \mid+\int_{\mathcal{S}} \phi\left(\nabla y(\tilde{x}) \mid b(\tilde{x}), \theta, \theta_{T}(\tilde{x})\right) d \tilde{x}-\int_{\mathcal{S}} \sigma \cdot(\nabla y(\tilde{x}) \mid b(\tilde{x})) d \tilde{x} \\
= & \left.\kappa \sum_{e \subset \mathcal{S}}\left|\llbracket(\nabla y \mid \sqrt{2} b) \rrbracket_{e}\right||e|+\left.\sum_{K \in \tau} \phi\left(\nabla y \mid b, \theta, \theta_{T}\right)\right|_{K}\right)|K|-\left.\sum_{K \in \tau} \sigma \cdot(\nabla y(\tilde{x}) \mid b(\tilde{x}))\right|_{K}|K|,
\end{aligned}
$$

where $|K|$ is the area of the element $K$ and

$$
|\llbracket(\nabla y \mid \sqrt{2} b) \rrbracket e|=\left(\left|\llbracket \nabla y \rrbracket_{e}\right|^{2}+2\left|\llbracket b \rrbracket_{e}\right|^{2}\right)^{1 / 2} \text {. }
$$

\section{The Elastic Energy Density}

To model the first-order cubic to tetragonal structural phase transformation at the transformation temperature, $\theta_{T}$, we shall assume that at temperatures $\theta>\theta_{T}$ the free energy density, $\phi\left(F, \theta, \theta_{T}\right)$, as a function of the deformation gradient $F \in \mathbb{R}_{+}^{3 \times 3}$ is minimized on the group of proper rotations, $\mathrm{SO}(3)$; and that for temperatures $\theta<\theta_{T}$ the free energy density as a function of the deformation gradient $F \in \mathbb{R}_{+}^{3 \times 3}$ is minimized on

$$
\mathcal{M}=\mathrm{SO}(3) U_{1} \cup \mathrm{SO}(3) U_{2} \cup \mathrm{SO}(3) U_{3},
$$

where

$$
U_{1}=\left(\begin{array}{ccc}
\gamma & 0 & 0 \\
0 & \alpha & 0 \\
0 & 0 & \alpha
\end{array}\right), \quad U_{2}=\left(\begin{array}{ccc}
\alpha & 0 & 0 \\
0 & \gamma & 0 \\
0 & 0 & \alpha
\end{array}\right), \quad U_{3}=\left(\begin{array}{ccc}
\alpha & 0 & 0 \\
0 & \alpha & 0 \\
0 & 0 & \gamma
\end{array}\right)
$$


We chose the transformation stretches [14]

$$
\alpha=0.9837, \quad \gamma=1.0260 .
$$

Following [14], the free energy density $\phi\left(F, \theta, \theta_{T}\right)$ is defined in terms of the right Cauchy-Green strain, $C=F^{T} F \in \mathbb{S}_{+}^{3 \times 3}$ (where $\mathbb{S}_{+}^{3 \times 3}$ is the space of symmetric positive definite matrices), to be

$$
\phi\left(F, \theta, \theta_{T}\right)=\min \left\{W_{A}(C, \theta), W_{M}(C, \theta)+\eta\left(\theta, \theta_{T}\right)\right\},
$$

where $W_{A}(C, \theta)$ and $W_{M}(C, \theta)$ are cubic and tetragonal elastic free energy densities, respectively, normalized to be zero only at their respective energy wells $\mathrm{SO}(3)$ and $\mathcal{M}$, and $\eta\left(\theta, \theta_{T}\right)$ is the free energy difference between the cubic and tetragonal phases. This free energy difference between the cubic and tetragonal phases, $\eta\left(\theta, \theta_{T}\right)$, satisfies

$$
\eta\left(\theta, \theta_{T}\right)=\left\{\begin{aligned}
W_{A}\left(U_{1}^{2}, A_{f}\right) \frac{\theta-\theta_{T}}{A_{f}-\theta_{T}} & \text { for } \theta_{T} \leq \theta<\theta_{1}, \\
-W_{M}\left(I, M_{f}\right) \frac{\theta-\theta_{T}}{M_{f}-\theta_{T}} & \text { for } \theta_{0}<\theta<\theta_{T},
\end{aligned}\right.
$$

for $A_{f}$ and $M_{f}$ such that $\theta_{0}<M_{f}<\theta_{T}<A_{f}<\theta_{1}$. The free energy difference, $\eta\left(\theta, \theta_{T}\right)$, is a continuous, piecewise linear function such that the local minimum corresponding to the tetragonal phase $(F \in \mathcal{M})$ disappears at $\theta=A_{f}$, and the local minimum corresponding to the cubic phase $(F \in \mathrm{SO}(3))$ disappears at $\theta=M_{f}$. In physical measurements, $A_{f}$ is the temperature at which an unloaded martensitic crystal has fully transformed to austenite (the high-temperature phase) during heating, and $M_{f}$ is the temperature at which an unloaded martensitic crystal has fully transformed to martensite (the low-temperature phase) during cooling.

We assume that the crystal has an average composition $\bar{c}=|\mathcal{S}|^{-1} \int_{\mathcal{S}} c(\tilde{x}) d \tilde{x}$ that corresponds to a transformation temperature $\bar{\theta}_{T}$, and we model the transformation temperature $\theta_{T}(\tilde{x})$ by independent normally distributed random variables $\left.\theta_{T}\right|_{K}$, indexed by the triangles $K \in \tau$, with mean

$$
\bar{\theta}_{T}=270 \mathrm{~K}
$$

and standard deviation $\theta_{s d}>0$ given by (7.1). Hence, $\theta_{T} \in \mathcal{P}_{0}(\tau)$, and we obtain a value for $\left.\theta_{T}\right|_{K}$ on each triangle $K \in \tau$ by using a normally distributed pseudo-random number generator with mean $\bar{\theta}_{T}$ and standard deviation $\theta_{s d}$.

The other transformation temperatures $A_{f}$ and $M_{f}$ are then defined as piecewise constant functions with respect to the triangles $K$ in the triangulation $\tau$ by

$$
A_{f}(\tilde{x})=\theta_{T}(\tilde{x})+5 \mathrm{~K} \quad \text { and } \quad M_{f}(\tilde{x})=\theta_{T}(\tilde{x})-15 \mathrm{~K} .
$$

It follows from (6.5) and (6.6) that $A_{f}(\tilde{x})$ and $M_{f}(\tilde{x})$ have standard deviation $\sigma=25 \mathrm{~K}$ and mean values

$$
\bar{A}_{f}=275 \mathrm{~K} \quad \text { and } \quad \bar{M}_{f}=255 \mathrm{~K} .
$$

In [14], we constructed the following austenitic free energy $W_{A}(C, \theta)$ having cubic symmetry:

$$
\begin{gathered}
W_{A}(C, \theta)=a(\theta)\left[\left(C_{11}-C_{22}\right)^{2}+\left(C_{22}-C_{33}\right)^{2}+\left(C_{11}-C_{33}\right)^{2}\right] \\
+b(\theta)(\operatorname{tr} C-3)^{2}+c(\theta)\left(C_{12}^{2}+C_{23}^{2}+C_{13}^{2}\right)
\end{gathered}
$$

where $C_{i j} \in \mathbb{R}$ are the matrix elements of the right Cauchy-Green strain $C=F^{T} F \in \mathbb{S}_{+}^{3 \times 3}$ and the elastic moduli satisfy

$$
a(\theta) \approx 0.0185 \tilde{\theta} \mathrm{GPa}, \quad b(\theta) \approx(17.2-0.0196 \tilde{\theta}) \mathrm{GPa}, \quad c(\theta) \approx(36.3+0.005 \tilde{\theta}) \mathrm{GPa},
$$

for

$$
\tilde{\theta}=\max \left\{\theta-M_{s}, 0\right\}
$$


with $M_{s}$ such that $M_{f}<M_{s}<\theta_{T}$ being the temperature at which one of the elastic moduli of the cubic phase converges to zero or "softens." We take

$$
M_{s}(\tilde{x})=\theta_{T}(\tilde{x})-5 \mathrm{~K}
$$

so $M_{s}$ has standard deviation $\theta_{s d}$ and mean value

$$
\bar{M}_{s}=265 \mathrm{~K} .
$$

In our model, we have that $\theta_{T}(\tilde{x})=\left(M_{s}(\tilde{x})+A_{f}(\tilde{x})\right) / 2$ as proposed in [39]. The moduli were chosen to fit experimental data for $\mathrm{Fe}_{70} \mathrm{Pd}_{30}[36]$.

We constructed a martensitic free energy density $W_{M_{3}}(C, \theta)$ as a function of $C$ by

$$
\begin{aligned}
W_{M_{3}}(C, \theta)=d & {\left[\left(C_{11}-\alpha^{2}\right)^{2}+\left(C_{22}-\alpha^{2}\right)^{2}\right]+e\left(C_{33}-\gamma^{2}\right)^{2}+f\left(C_{11}-\alpha^{2}\right)\left(C_{22}-\alpha^{2}\right) } \\
& +g\left(C_{33}-\gamma^{2}\right)\left(C_{11}+C_{22}-2 \alpha^{2}\right)+h_{1}\left(C_{23}^{2}+C_{13}^{2}\right)+h_{2} C_{12}^{2},
\end{aligned}
$$

where in terms of the elastic moduli $\mathbb{C}_{i j}^{M}$ in the Voigt notation [14], we have

$$
\begin{aligned}
& d=\mathbb{C}_{11}^{M} / 8 \alpha^{4}, \quad e=\mathbb{C}_{33}^{M} / 8 \gamma^{4}, \quad f=\mathbb{C}_{12}^{M} / 4 \alpha^{4}, \\
& g=\mathbb{C}_{13}^{M} / 4(\alpha \gamma)^{2}, \quad h_{1}=\mathbb{C}_{44}^{M} / 2(\alpha \gamma)^{2}, \quad h_{2}=\mathbb{C}_{66}^{M} / 2 \alpha^{4} .
\end{aligned}
$$

We construct the free energy densities $W_{M_{1}}(C, \theta)$ and $W_{M_{2}}(C, \theta)$, minimized at $U_{1}^{2}$ and $U_{2}^{2}$, respectively, by symmetry from

$$
\begin{array}{ll}
W_{M_{1}}(C, \theta)=W_{M_{3}}\left(R\left(\frac{\pi}{2}, e_{2}\right)^{T} C R\left(\frac{\pi}{2}, e_{2}\right), \theta\right) & \text { for all } C \in \mathbb{S}_{+}^{3 \times 3}, \\
W_{M_{2}}(C, \theta)=W_{M_{3}}\left(R\left(\frac{\pi}{2}, e_{1}\right)^{T} C R\left(\frac{\pi}{2}, e_{1}\right), \theta\right) & \text { for all } C \in \mathbb{S}_{+}^{3 \times 3},
\end{array}
$$

where $R\left(\frac{\pi}{2}, e_{i}\right) \in \mathcal{G}$ denotes the rotation of $\frac{\pi}{2}$ radians about the orthogonal basis vectors $e_{i} \in \mathbb{R}^{3}$.

By the symmetry in our construction of the free energy densities $W_{M_{i}}(C, \theta)$, there exists $\nu>0$, independent of $i$ and $j$, such that

$$
W_{M_{i}}\left(U_{j}^{2}, \theta\right)=\nu \quad \text { for } i \neq j .
$$

We then have that the quartic function

$$
W_{M}^{i}(C, \theta)=\frac{1}{2 \nu} W_{M_{i}}(C, \theta) \sum_{j=1}^{3}\left[W_{M_{j}}(C, \theta)\left(1-\delta_{i j}\right)\right]
$$

(where $\delta_{i j}$ is the Kronecker delta) is nonnegative, equals 0 only at the variant $U_{i}^{2}$, and matches the prescribed tetragonal elastic moduli at $U_{i}^{2}$. We then define the martensitic elastic free energy density $W_{M}(C, \theta)$ by

$$
W_{M}(C, \theta)=\min _{1 \leq i \leq 3} W_{M}^{i}(C, \theta) .
$$

In our computations, we used the following moduli in GPa:

$$
\mathbb{C}_{11}^{M}=150, \quad \mathbb{C}_{33}^{M}=150, \quad \mathbb{C}_{12}^{M}=130, \quad \mathbb{C}_{13}^{M}=130, \quad \mathbb{C}_{44}^{M}=70, \quad \mathbb{C}_{66}^{M}=75 .
$$




\section{The Numerical Experiment}

We computed solutions to the temperature and loading cycle described in Section 1 by following a path of local minima of the thin film energy $\overline{\mathcal{E}}_{0}(y, b ; \theta, \sigma)$ for $(y, b) \in \overline{\mathcal{A}}_{\tau}$ as $\theta, \sigma_{1}$, and $\sigma_{2}$ are varied. We set the surface energy parameter $\kappa=10^{-4} \mathrm{GPa} \cdot L$ where $L$ is the length of the crystal in meters which has been scaled to $\mathcal{S}=(-1 / 2,1 / 2) \times(-1 / 2,1 / 2)$. Since the elastic moduli have been taken to be of the order $100 \mathrm{GPa}$, the dimensionless surface energy $\kappa /(\mathbb{C} L)$ is of the order $10^{-6}$.

Our finite element mesh was constructed by dividing the square computational domain $\mathcal{S}=$ $(-1 / 2,1 / 2) \times(-1 / 2,1 / 2)$ into $N \times N$ congruent squares with sides of length $h=1 / N$. We further subdivided each of these squares into four triangles by the diagonals of the square. Piecewise affine deformations constrained to the energy-minimizing martensitic variants (6.1) have piecewise constant gradients $(\nabla y \mid b)$ that can be discontinuous across the diagonals $[9,11,33]$, so this grid allows an efficient approximation of a solution with microstructure. We present results on meshes with $N=50$ and $N=100$. Numerical analysis and computational experiments have shown that similar results can be obtained for finer meshes that are not oriented to the microstructure [30,33,34].

We set the standard deviation of the transformation temperatures to be

$$
\theta_{s d}=\frac{N}{2} \mathrm{~K}
$$

so that $\theta_{s d}=25 \mathrm{~K}$ for our simulations with $N=50$ and $\theta_{s d}=50 \mathrm{~K}$ for our simulations with $N=100$. The scaling of (7.1) with respect to $N$ is consistent with assuming that $\theta_{T}(\tilde{x})$ depends linearly on the composition $c(\tilde{x})$ and assuming that the average composition $\left.c\right|_{K}$ on the triangles $K \in \tau$ are independent normally distributed random variables indexed by the triangles $K \in \tau$.

We used increments of $1 \mathrm{~K}$ in our quasi-static continuation during the cooling of the thin film from $315 \mathrm{~K}$ to $215 \mathrm{~K}$ and then during the heating from $215 \mathrm{~K}$ to $315 \mathrm{~K}$. We used increments of 0.003 GPa for the stress $\sigma_{1}$ during the uniaxial loading, biaxial loading cycle, and uniaxial unloading. The continuation is given by the following path (in units $\mathrm{K}$ for the temperature $\theta$ and GPa for the stress $\sigma)$ :

1. Cooling: $\theta_{\ell}=315-\ell, \sigma_{1, \ell}=0, \sigma_{2, \ell}=0$ for $\ell=0, \ldots, 100$.

2. Uniaxial loading: $\theta_{\ell}=215, \sigma_{1, \ell}=0.003(\ell-101), \sigma_{2, \ell}=0$ for $\ell=101, \ldots, 201$.

3. Biaxial loading cycle:

a. $\theta_{\ell}=215, \sigma_{1, \ell}=0.3-0.003(\ell-202), \sigma_{2, \ell}=0.003(\ell-202)$ for $\ell=202, \ldots, 302$.

b. $\theta_{\ell}=215, \sigma_{1, \ell}=0.003(\ell-303), \sigma_{2, \ell}=0.3-0.003(\ell-303)$ for $\ell=303, \ldots, 403$.

4. Unaxial unloading: $\theta_{\ell}=215, \sigma_{1, \ell}=0.3-0.003(\ell-404), \sigma_{2, \ell}=0$ for $\ell=404, \ldots, 504$.

5. Heating: $\theta_{\ell}=215+(\ell-505), \sigma_{1, \ell}=0, \sigma_{2, \ell}=0$ for $\ell=505, \ldots, 605$.

6. Cooling: $\theta_{\ell}=315-(\ell-606), \sigma_{1, \ell}=0, \sigma_{2, \ell}=0$ for $\ell=606, \ldots, 706$.

7. Heating: $\theta_{\ell}=215+(\ell-707), \sigma_{1, \ell}=0, \sigma_{2, \ell}=0$ for $\ell=707, \ldots, 807$.

8. Cooling: $\theta_{\ell}=315-(\ell-808), \sigma_{1, \ell}=0, \sigma_{2, \ell}=0$ for $\ell=808, \ldots, 908$.

9. Heating: $\theta_{\ell}=215+(\ell-909), \sigma_{1, \ell}=0, \sigma_{2, \ell}=0$ for $\ell=909, \ldots, 1009$.

We assume that the rate of cooling, loading, unloading, and heating is slow enough so that the film is always in elastic equilibrium at a constant temperature and load.

Given an initial deformation $\left(y_{0}, b_{0}\right)$, we determine the deformation $\left(y_{\ell}, b_{\ell}\right) \in \overline{\mathcal{A}}_{\tau}$ for $\ell=$ $1, \ldots, 1009$ by computing a local minimum for the energy $\overline{\mathcal{E}}^{0}\left(y, b ; \theta_{\ell}, \sigma_{\ell}\right)$ from the Polak-Ribière 
conjugate gradient method $[10,38]$ with initial iterate

$$
\left(y_{\ell}^{[0]}, b_{\ell}^{[0]}\right)=\left(y_{\ell-1}, b_{\ell-1}\right) .
$$

After the conjugate gradient iterations have converged to an acceptable tolerance after $M$ iterations, we set

$$
\left(y_{\ell}, b_{\ell}\right)=\left(y_{\ell}^{[M]}, b_{\ell}^{[M]}\right) .
$$

We can compute the gradient $\left(g_{1}, g_{2}\right) \in \overline{\mathcal{A}}_{\tau}=\overline{\mathcal{P}}_{1}(\tau) \times \mathcal{P}_{0}(\tau)$ of the energy $\overline{\mathcal{E}}^{(0)}(y, b ; \theta, \sigma)$ with respect to $(y, b) \in \overline{\mathcal{A}}_{\tau}$ by the $L^{2}(S)$-projection of the first variation of the energy

$$
\int_{S}\left(g_{1} \tilde{y}+g_{2} \tilde{b}\right)=\delta \overline{\mathcal{E}}^{(0)}(y, b ; \theta, \sigma)(\tilde{y}, \tilde{b}) \quad \text { for all }(\tilde{y}, \tilde{b}) \in \overline{\mathcal{A}}_{\tau}
$$

In the above equation, $\delta \overline{\mathcal{E}}^{(0)}(y, b ; \theta, \sigma)(\tilde{y}, \tilde{b})$ is the Gâteaux derivative of the energy in the direction $(\tilde{y}, \tilde{b}) \in \overline{\mathcal{A}}_{\tau}$. It can then be seen that the constraint $\int_{S} y=0$ is preserved by the conjugate gradient iterates since $\int_{S} g_{1}=0$ for all of the gradients.

The simulation starts with the film at $315 \mathrm{~K}$ in the nearly flat cubic (high-temperature) state given by

$$
\begin{array}{ll}
y_{0}\left(x_{1}, x_{2}\right)=\left(x_{1}, x_{2}, \delta y_{0}\right) & \text { for all } \tilde{x}=\left(x_{1}, x_{2}\right) \in \mathcal{S}, \\
b_{0}\left(x_{1}, x_{2}\right)=(0,0,1) & \text { for all } \tilde{x}=\left(x_{1}, x_{2}\right) \in \mathcal{S},
\end{array}
$$

where a small perturbation of the perfectly flat state is given by $\delta y_{0}$. Since the temperatures at which the high-temperature phase loses stability on the triangles $K \in \tau$ are independent normally distributed random variables $\left.M_{f}\right|_{K}$ with mean $\bar{M}_{f}=255 \mathrm{~K}$ and standard deviation $\theta_{s d}$, we expect that $\left.M_{f}\right|_{K}<315 \mathrm{~K}$ for nearly all triangles $K \in \tau$. Thus, we can expect that the Polak-Ribière conjugate gradient method will give a state $\left(y_{1}, b_{1}\right)$ that is close to the energy-minimizing perfectly flat state

$$
\begin{array}{ll}
y_{0}\left(x_{1}, x_{2}\right)=\left(x_{1}, x_{2}, 0\right) & \text { for all } \tilde{x}=\left(x_{1}, x_{2}\right) \in \mathcal{S}, \\
b_{0}\left(x_{1}, x_{2}\right)=(0,0,1) & \text { for all } \tilde{x}=\left(x_{1}, x_{2}\right) \in \mathcal{S},
\end{array}
$$

while retaining some pertubation in $y_{3}$ that will potentially allow a larger out-of-plane transformation and attainment of lower energy as the film is cooled.

We obtain the components of $\delta y_{0}$ at the nodes of the triangulation by a normally distributed pseudo-random number generator with mean 0.0 and standard deviation $0.005 h$, where $h=1 / N$. Since the values of the computed pseudo-random $\delta y_{0}$ at the mesh points are independent, the standard deviation of the derivatives of $\delta y_{0}$ are $0.005 \sqrt{2}$ and $0.005 \sqrt{6}$ depending on the orientation of the triangle.

As in [10], we actually computed the gradients used in the conjugate gradient iterations by replacing the "mass" matrix on the left-hand side of (7.3) by the identity matrix with respect to the classical Lagrangian shape functions for the continuous, piecewise linear finite element space [17]. With this replacement, the mean-zero property $\int_{S} y_{\ell}=0$ is only approximately satisfied, but we can replace $y_{\ell}$ by $y_{\ell}-|S|^{-1} \int_{S} y_{\ell}$ to regain the mean-zero property at any time in the iteration without affecting the computation since the energy (4.3) is invariant with respect to the translation $y \rightarrow y+c$ for $c \in \mathbb{R}^{3}$.

\section{Numerical Results AND Interpretation}

In Figures 1-3 and Tables 1-3, we present the results of the temperature and loading cycle described in Section 7. The coloring scheme used to exhibit the deformation gradient $\left.(\nabla y \mid b)\right|_{K}$ is based on its distance to the nearest phase or variant [33]. Elements with deformation gradient $\left.(\nabla y \mid b)\right|_{K}$ near the cubic phase $F \in \mathrm{SO}(3)$ are colored a shade of grey and elements with deformation 
gradient $\left.(\nabla y \mid b)\right|_{K}$ near the tetragonal variants are colored shades of red for $F \in \operatorname{SO}(3) U_{1}$, shades of yellow for $F \in \mathrm{SO}(3) U_{2}$, and shades of blue for $F \in \mathrm{SO}(3) U_{3}$.

The phase transformation predicted by our model during the cooling and heating phases has been studied in [14]. The energy density that we used for the computations reported in this paper allows the loss of stability of both of the phases at sufficiently high or low temperatures, compositional variation, and elastic softening $[11,14]$.

After the first cooling, the film reaches the twinned state in the upper left corner of Figure 1. The volume fractions of the red variant $\left(\mathrm{SO}(3) U_{1}\right)$ and the yellow variant $\left(\mathrm{SO}(3) U_{2}\right)$ are approximately equal. We then observe in the first row of Figure 1 (see also Table 1) the growth of the volume fraction of the red variant as the film undergoes an increasing uniaxial tensile load $\sigma_{1}$ on the planes $x_{1}=-1 / 2$ and $x_{1}=1 / 2$. In the second row, we observe the growth of the volume fraction of the yellow variant during the first phase of the biaxial loading cycle as the tensile load $\sigma_{1}$ on the planes $x_{1}=-1 / 2$ and $x_{1}=1 / 2$ decreases and the tensile load $\sigma_{2}$ on the planes $x_{2}=-1 / 2$ and $x_{2}=1 / 2$ increases while maintaining the constraint $\sigma_{1}+\sigma_{2}=0.3 \mathrm{GPa}$. In the third row, we observe the growth of the volume fraction of the red variant during the second phase of the biaxial loading cycle as the load $\sigma_{1}$ increases and the load $\sigma_{2}$ decreases while maintaining the constraint $\sigma_{1}+\sigma_{2}=0.3$ $\mathrm{GPa}$. In the fourth row, we find that the film remains nearly completely in the "red" variant as the film is unloaded.

These results can be understood theoretically by noting that the elastic energy density of the crystal is equal in the red variant $\left(\mathrm{SO}(3) U_{1}\right)$ and the yellow variant $\left(\mathrm{SO}(3) U_{2}\right)$ since

$$
\phi\left(U_{1}, \theta, \theta_{T}\right)=\phi\left(U_{2}, \theta, \theta_{T}\right),
$$

and the loading potential energy density $-\sigma \cdot F$ is minimized on $\operatorname{SO}(3) U_{1} \cup \operatorname{SO}(3) U_{2}$ at $U_{1}$ if $\sigma_{1}>\sigma_{2}$ and at $U_{2}$ if $\sigma_{1}<\sigma_{2}$. More generally, we can expect the loading energy density during the biaxial loading cycle to be approximately of the form

$$
-\sigma \cdot\left[\lambda R_{1} U_{1}+(1-\lambda) R_{2} U_{2}\right]
$$

where $0<\lambda<1$ and $R_{1}, R_{2} \in \mathrm{SO}(3)$. We then can expect that $\lambda \rightarrow 0$ and $R_{2} \rightarrow I$ as $\sigma_{1}-\sigma_{2}$ decreases and that $\lambda \rightarrow 1$ and $R_{1} \rightarrow I$ as $\sigma_{1}-\sigma_{2}$ increases during the biaxial loading cycle.

In Figures 2-3 and Tables 2-3, we can observe the effect of the first loading cycle on the state of the film after cooling from $315 \mathrm{~K}$ to $215 \mathrm{~K}$. We see in the left figure of the third row of Figures $2-3$ that the film is nearly completely in the red variant after being heated to $315 \mathrm{~K}$ and then cooled to $215 \mathrm{~K}$ following the first loading cycle. We observe in the right figure of the first row of Figures $2-3$ that the volume fractions of the red and yellow variants were nearly equal following the initial cooling from $315 \mathrm{~K}$ to $215 \mathrm{~K}$. After another heating and cooling cycle, we find in the left figure of the fourth row in Figures 2-3 that the film continues to return to a nearly uniform "red" variant state. The return to the "red" variant state is not as complete for the coarser mesh $N=50$ as for the finer mesh $N=100$. One source for the difference is from sampling the transformation temperatures $\theta_{T}(\tilde{x})$ for $N=50$ and $N=100$ by a normally distributed pseudo-random number generator with standard deviation (7.1) for $\left.\theta_{T}\right|_{K}$, indexed by the triangles $K \in \tau$. We hope that a complete error analysis can be obtained to explain this.

During the biaxial loading cycle, the deformation $(y, b)$ evolves through a sequence of metastable states for the energy $\overline{\mathcal{E}}^{0}\left(y, b ; \theta_{\ell}, \sigma_{\ell}\right)$ as the loading stress $\sigma$ is varied. We note that the red $\left(\mathrm{SO}(3) U_{1}\right)$ and yellow $\left(\mathrm{SO}(3) U_{2}\right)$ variants can lose stability for the Gibbs free energy density, the sum of the elastic energy density and the loading potential energy density

$$
\phi\left(F, \theta, \theta_{T}\right)-\sigma \cdot F,
$$

as the loading stress $\sigma$ is cycled. 


\begin{tabular}{|c|c|c|}
\hline$\sigma_{1}-\sigma_{2}$ & red variant & yellow variant \\
\hline 0.00 & 41.47 & 24.94 \\
0.15 & 90.76 & 1.50 \\
0.3 & 99.82 & 0.00 \\
0.00 & 98.48 & 0.00 \\
-0.3 & 0.00 & 99.85 \\
0.00 & 0.00 & 98.50 \\
0.3 & 99.84 & 0.00 \\
0.15 & 99.56 & 0.00 \\
0.00 & 94.08 & 0.00 \\
\hline
\end{tabular}

TABle 1. $(N=100)$ Percent volume fraction for the red and yellow variant during the biaxial loading cycle shown in Figure 1.

\begin{tabular}{|c|c|c|}
\hline temperature K & red variant & yellow variant \\
\hline 315 & 0.00 & 0.00 \\
215 & 36.82 & 36.17 \\
215 & 99.99 & 0.00 \\
315 & 0.00 & 0.00 \\
215 & 79.22 & 9.47 \\
315 & 0.00 & 0.00 \\
215 & 80.47 & 8.55 \\
315 & 0.00 & 0.00 \\
\hline
\end{tabular}

TABle 2. $(N=50)$ Percent volume fraction for the red and yellow variant during the cooling and heating cycles shown in Figure 2.

\begin{tabular}{|c|c|c|}
\hline temperature K & red variant & yellow variant \\
\hline 315 & 0.00 & 0.00 \\
215 & 41.47 & 24.94 \\
215 & 94.08 & 0.00 \\
315 & 0.00 & 0.00 \\
215 & 93.45 & 0.00 \\
315 & 0.00 & 0.00 \\
215 & 93.45 & 0.00 \\
315 & 0.00 & 0.00 \\
\hline
\end{tabular}

TABle 3. $(N=100)$ Percent volume fraction for the red and yellow variant during the cooling and heating cycles shown in Figure 3.

At the end of the cycle with $\sigma_{1}=0.3 \mathrm{GPa}$ and $\sigma_{2}=0.0 \mathrm{GPa}$ the film is nearly completely in the red variant state. During the next heating stage, the film transforms nearly completely to the high-temperature phase, but remains in a metastable state with a path back to the red variant phase during the subsequent cooling. 

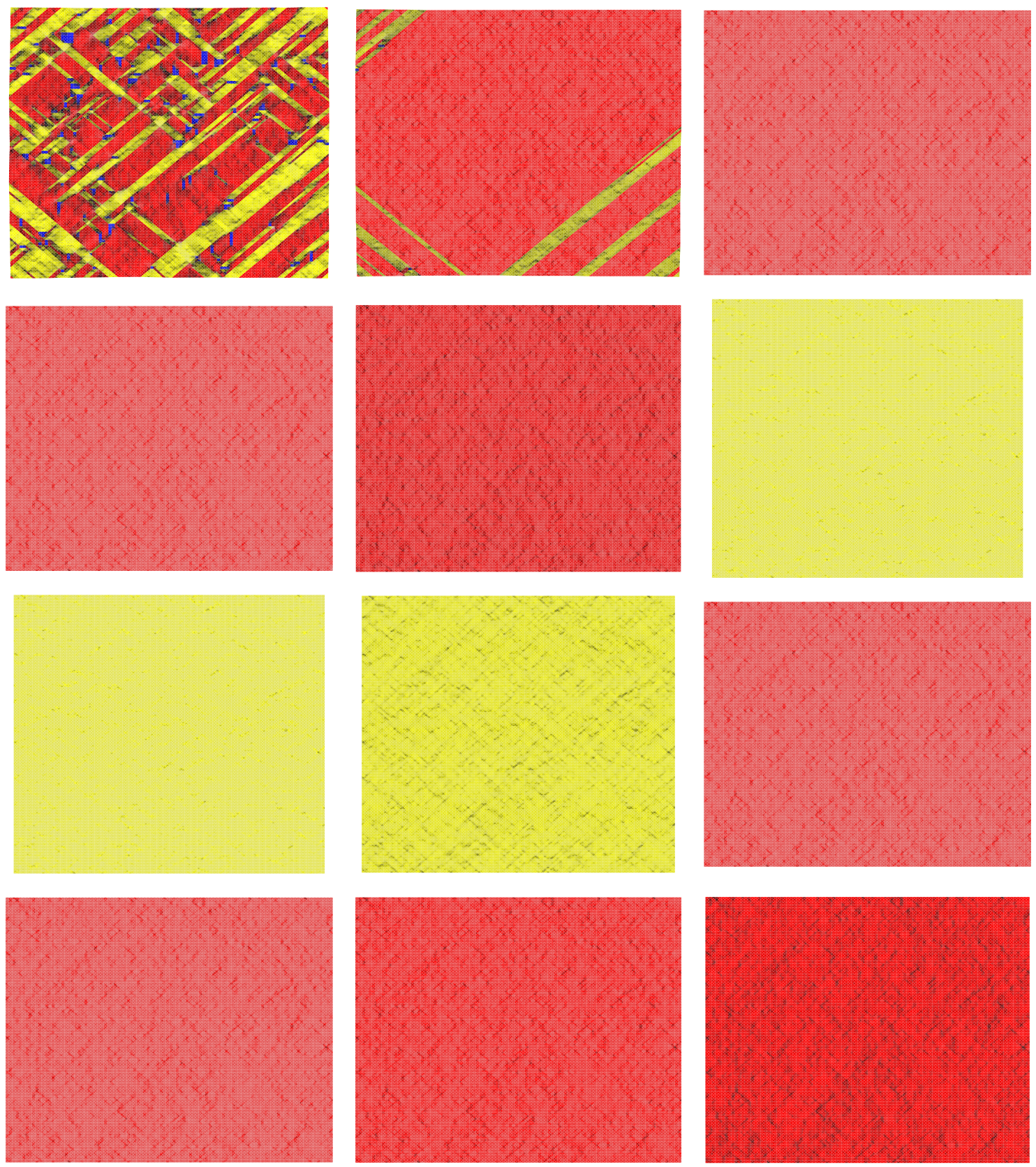

Figure 1. $(N=100)$ First row: Uniaxial tensile loading $\sigma_{1}=0.0,0.15,0.3 \mathrm{GPa}$ with $\sigma_{2}=0.0 \mathrm{GPa}$ on the planes $x_{1}=-1 / 2$ and $x_{1}=1 / 2$ after the first cooling. Second row: Biaxial loading $\sigma_{1}=0.3,0.15,0.0 \mathrm{GPa}$ for $\sigma_{1}+\sigma_{2}=0.3 \mathrm{GPa}$. Third row: Biaxial loading $\sigma_{1}=0.0,0.15,0.3 \mathrm{GPa}$ for $\sigma_{1}+\sigma_{2}=0.3 \mathrm{GPa}$. Fourth row: Uniaxial tensile unloading $\sigma_{1}=0.3,0.15,0.0 \mathrm{GPa}$ with $\sigma_{2}=0.0 \mathrm{GPa}$. 

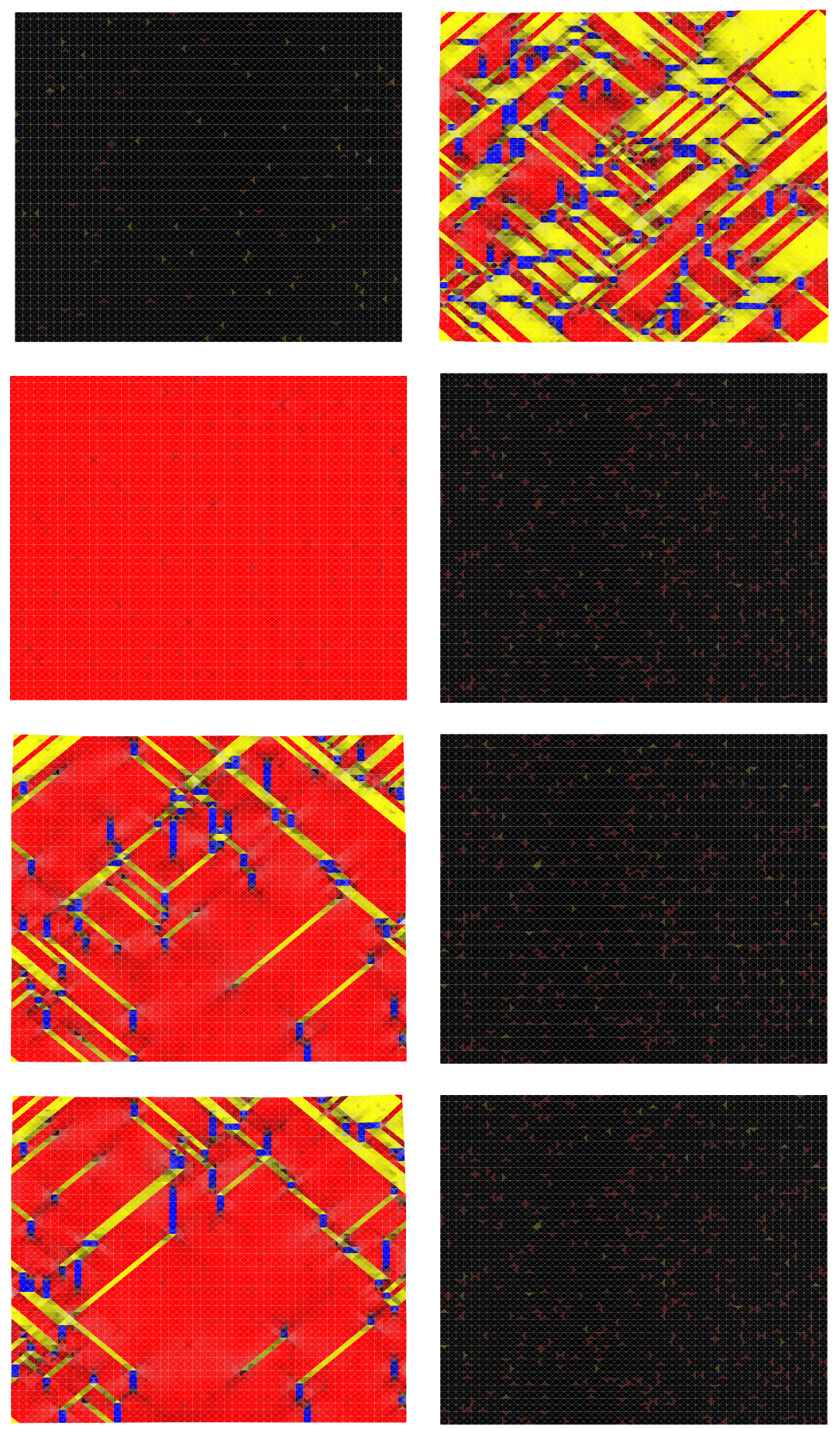

Figure 2. $(N=50)$ First row: Initial state at $315 \mathrm{~K}$ (left) and after cooling to $215 \mathrm{~K}$ (right). Second row: At $215 \mathrm{~K}$ after loading cycle (left) and then heating to $315 \mathrm{~K}$ (right). Third row: After cooling to $215 \mathrm{~K}$ (left) and heating to $315 \mathrm{~K}$ (right). Fourth row: After cooling again to $215 \mathrm{~K}$ (left) and heating to $315 \mathrm{~K}$ (right). 

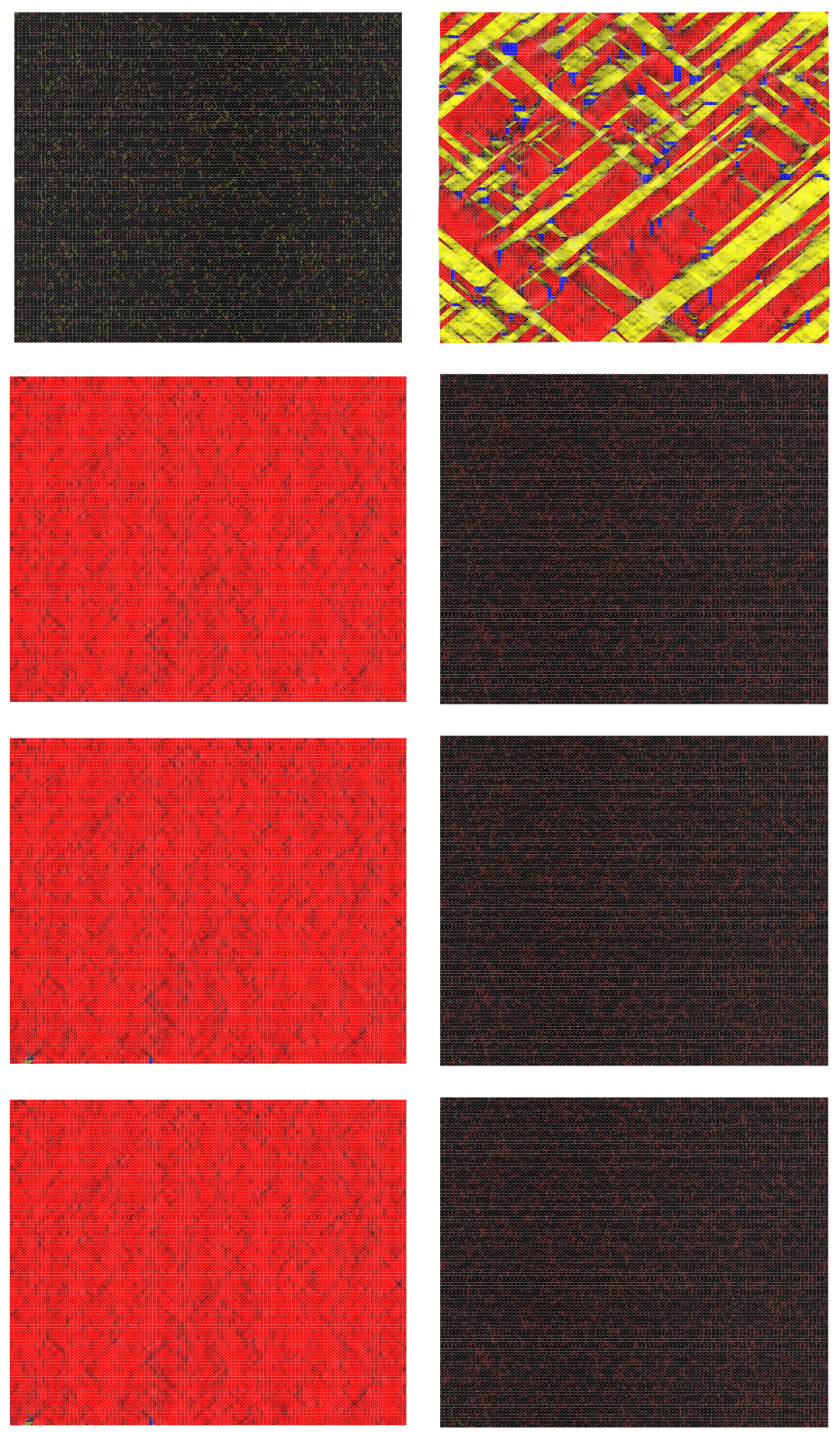

Figure 3. $(N=100)$ First row: Initial state at $315 \mathrm{~K}$ (left) and after cooling to $215 \mathrm{~K}$ (right). Second row: At $215 \mathrm{~K}$ after loading cycle (left) and then heating to $315 \mathrm{~K}$ (right). Third row: After cooling to $215 \mathrm{~K}$ (left) and heating to $315 \mathrm{~K}$ (right). Fourth row: After cooling again to $215 \mathrm{~K}$ (left) and heating to $315 \mathrm{~K}$ (right). 


\section{REFERENCES}

[1] R. Abeyaratne, C. Chu, and R. James. Kinetics of materials with wiggly energies: Theory and application to the evolution of twinning microstructures in a Cu-Al-Ni shape memory alloy. Philosophical Magazine A, 73:457-497, 1996.

[2] R. Adams. Sobolev Spaces. Academic Press, New York, 1975.

[3] M. Arndt. Modelling and numerical simulation of martensitic transformation. In W. L. Wendland and M. Efendiev, editors, Analysis and Simulation of Multifield Problems, volume 12 of Lecture Notes in Applied and Computational Mechanics, pages 59-65. Springer, 2003.

[4] M. Arndt, M. Griebel, and T. Roubíček. Modelling and numerical simulation of martensitic transformation in shape memory alloys. Continuum Mechanics and Thermodynamics, 15(5):463-485, 2003.

[5] J. Ball, C. Chu, and R. James. Hysteresis during stress-induced variant rearrangement. J. de Physique IV, C8:245-251, 1995.

[6] J. M. Ball and R. D. James. Fine phase mixtures as minimizers of energy. Arch. Ration. Mech. Anal., 100(1):13$52,1987$.

[7] J. M. Ball and R. D. James. Proposed experimental tests of a theory of fine microstructure and the two-well problem. Phil. Trans. R. Soc. Lond. A, 338:389-450, 1992.

[8] S. Bartels, C. Carstensen, K. Hackl, and U. Hoppe. Effective relaxation for microstructure simulations: algorithms and applications. Comput. Methods Appl. Mech. Engrg., 193(48-51):5143-5175, 2004.

[9] K. Bhattacharya and R. D. James. A theory of thin films of martensitic materials with applications to microactuators. J. Mech. Phys. Solids, 47(3):531-576, 1999.

[10] P. Bělík, T. Brule, and M. Luskin. On the numerical modeling of deformations of pressurized martensitic thin films. Math. Model. Numer. Anal., 35:525-548, 2001.

[11] P. Bělík and M. Luskin. A computational model for the indentation and phase transformation of a martensitic thin film. J. Mech. Phys. Solids, 50:1789-1815, 2002.

[12] P. Bělík and M. Luskin. A total-variation surface energy model for thin films of martensitic crystals. Interfaces Free Bound., 4:71-88, 2002.

[13] P. Bělík and M. Luskin. A computational model for martensitic thin films with compositional fluctuation. Mathematical Models \& Methods in Applied Sciences, 14:1585-1598, 2004.

[14] P. Bělík and M. Luskin. Computational modeling of softening in a structural phase transformation. Multiscale Modeling \& Simulation, 3:764-781, 2004.

[15] P. Bělík and M. Luskin. The $\Gamma$-convergence of a sharp interface thin film model with non-convex elastic energy. SIAM J. Math. Anal., to appear.

[16] C. Carstensen and P. Plecháč. Numerical analysis of a relaxed variational model of hysteresis in two-phase solids. Math. Model. Numer. Anal., 35(5):865-878, 2001.

[17] P. Ciarlet. The Finite Element Method for Elliptic Problems. North-Holland, Amsterdam, 1978.

[18] B. Dacorogna. Direct methods in the calculus of variations. Springer-Verlag, Berlin, 1989.

[19] P. W. Dondl and J. Zimmer. Modeling and simulation of martensitic phase transitions with a triple point. J. Mech. Phys. Solids, 52(9):2057-2077, 2004.

[20] W. E and X. Li. Multiscale modeling of dynamics of solids at finite temperature. J. Mech. Phys. Solids, submitted.

[21] L. C. Evans and R. F. Gariepy. Measure Theory and Fine Properties of Functions. CRC Press, Boca Raton, FL, 1992.

[22] D. Gilbarg and N. S. Trudinger. Elliptic Partial Differential Equations of Second Order. Springer-Verlag, New York, 1998.

[23] E. Giusti. Minimal Surfaces and Functions of Bounded Variation. Birkhäuser Verlag, Basel-Boston, Mass., 1984.

[24] S. Kartha, J. A. Krumhansl, J. P. Sethna, and L. K. Wickham. Disorder-driven pretransitional tweed in martensitic transformations. Phys. Rev. B, 52:803-822, 1995.

[25] D. Kinderlehrer. Twinning in crystals II. In S. Antman, J. Ericksen, D. Kinderlehrer, and I. Müller, editors, IMA Volumes in Mathematics and Its Applications, vol. 3, pages 185-212, New York, 1987. Springer-Verlag.

[26] D. Kinderlehrer. Metastability and hysteresis in active materials. In Smart structures and materials 1997: mathematics and control in smart structures (San Diego, CA), volume 3039 of Proc. SPIE, pages 2-13. SPIE, Bellingham, WA, 1997.

[27] M. Kružík. Numerical approach to double well problems. SIAM J. Numer. Anal., 35:1833-1849, 1998.

[28] M. Kružík and F. Otto. A phenomenological model for hysteresis in polycrystalline shape memory alloys. Zeit. Angew. Math. Mech., 84:835-842, 2004. 
[29] B. Li. Approximation of martensitic microstructure with general homogeneous boundary data. J. Math. Anal. Appl., 266:451-467, 2002.

[30] B. Li and M. Luskin. Finite element analysis of microstructure for the cubic to tetragonal transformation. SIAM J. Numer. Anal., 35:376-392, 1998.

[31] Z. Li. Rotational transformation method and some numerical techniques for computing microstructures. Math. Models Methods Appl. Sci., 8:985-1002, 1998.

[32] Z. Li. Multiscale modelling and computation of microstructures in multi-well problems. Math. Models Methods Appl. Sci., 14:1343-1360, 2004.

[33] M. Luskin. On the computation of crystalline microstructure. Acta Numer., 5:191-258, 1996.

[34] M. Luskin. Computational modeling of microstructure. In Proceedings of the International Congress of Mathematicians, ICM 2002, Beijing, volume III, pages 707-716, 2002.

[35] G. D. Maso. An introduction to Г-convergence. Birkhäuser, 1993.

[36] S. Muto, R. Oshima, and F. Fujita. Elastic softening and elastic strain energy consideration in the FCC-FCT transformation of Fe-Pd alloys. Acta Metall. Mater., 38(4):685-694, 1990.

[37] S.-C. Ngan and L. Truskinovsky. Thermo-elastic aspects of dynamic nucleation. J. Mech. Phys. Solids, 50:11931229, 2002.

[38] J. Nocedal and S. Wright. Numerical Optimization. Springer-Verlag, 1999.

[39] K. Otsuka and C. M. Wayman. Shape Memory Materials. Cambridge University Press, Cambridge, UK, 1998.

[40] M. Pitteri and G. Zanzotto. Continuum models for twinning and phase transitions in crystals. Chapman and Hall, London, 1996.

[41] P. Plecháč and T. Roubíček. A visco-elasto-plastic model for phase transformations. Math. Meth. Appl. Sci., 25:1281-1298, 2000.

[42] A. Vainchtein. Dynamics of phase transitions and hysteresis in a viscoelastic Ericksen's bar on an elastic foundation. J. Elasticity, 57:243-280, 1999.

[43] J. Zimmer. Stored energy functions for phase transitions in crystals. Arch. Ration. Mech. Anal., 172(2):191-212, 2004.

Pavel Bělík, Department of Mathematics, University of St. Thomas, 2115 Summit Avenue, St. Paul, MN 55105, U.S.A.

E-mail address: pbelik@stthomas.edu

Mitchell Luskin, School of Mathematics, University of Minnesota, 206 Church Street SE, MinNEAPOLIS, MN 55455, U.S.A.

E-mail address: luskin@math.umn.edu 